\title{
Pro-Environmental Attitudes, Altruism, and COVID-19 Risk Management Behavior
}

\author{
Trisha R. Shrum, Sarah A. Nowak, Scott C. Merrill, John P. Hanley, Tung-Lin \\ Liu, Eric M. Clark, Asim Zia, and Christopher Koliba \\ University of Vermont, tshrum@uvm.edu, ORCID: 0000-0001-8660-155X
}

\begin{abstract}
Limiting the spread of COVID-19 during the pandemic is a collective action problem that calls on individuals to act, not just for their benefit but also for the benefit of others in their community. Many environmental problems, especially climate change, share this same characteristic. In this study, we use a survey instrument to examine how pro-environmental attitudes and two types of altruism relate to the tendency to follow social distancing guidelines and limit voluntary social exposure during the pandemic. We find that pro-environmental behaviors predict a feeling of moral obligation to reduce COVID-19 risk and a lower level of voluntary social exposure. Voluntary, individual-level altruism has no relationship with social exposure. These findings point to important insights about the nuances in altruistic behavior. These insights may lead to essential guidance for public health and environmental messaging that respects and leverages the differences in voluntary, individual-level altruism and collective altruism.
\end{abstract}

Keywords: COVID-19, risk behavior, pro-environmental attitudes, altruism

\section{Introduction}

The COVID-19 pandemic is an extraordinary global event whose mitigation has relied heavily on the will of individuals to act to protect the health of those around them. This is especially true in the United States where tightly enforced top-down 
mandates were largely delayed or avoided. Much of the public messaging around COVID-19 risk management guidelines has tried to leverage values of altruism and collectivism. Slogans or hashtags included "flatten the curve," "stay home save lives," and "wear a mask. save a life." Each of these calls on the individual to take an action to help those around them. This call to place the greater good above one's own desires shares many common threads with environmental movements that call on people to act to protect the environment even when they may incur a personal cost.

The desire to act in a way that benefits others is called prosocial motivation. There are four distinct forms of prosocial motivation based on the ultimate driver of that motivation: egoism (seeking benefits for the self), altruism (seeking benefits for another individual), collectivism (seeking benefits for a group), and principlism (seeking to uphold a moral principle) (Batson, Ahmad, \& Stocks, 2011). All four of these forms of prosocial motivation could drive social distancing behavior during the COVID-19 pandemic. One may make choices to avoid social contact because they want to avoid getting themselves sick (i.e. egoism), hope to keep their family members well (i.e. altruism), reduce the level of spread in their community (i.e. collectivism), and adhere to the principle of the golden rule (i.e. principlism). In this paper, we focus primarily on altruism as a potential driver of behavior, how altruistic motivations may overlap with pro-environmental worldviews, and look for new insights that may inform future work in both public health and environmental spaces.

An extensive literature that seeks to understand the characteristics that are common to those who hold pro-environmental attitudes has painted a fairly robust portrait of the prototypical environmentalist. Some of those common characteristics are potentially relevant for COVID-19 risk management behavior, specifically, selftranscendent values such as universalism (Schwartz, 1994), collectivism (Batson et al., 2011), and altruism (Heberlein, 1972; Menges, Schroeder, \& Traub, 2005; Popp, 2001; Stern, Kalof, Dietz, \& Guagnano, 1995).

One of the early connections between pro-environmental behavior and altruism relied on the logic that since environmental quality is a public good, contributing to the public good required a degree of altruism (Heberlein, 1972). This concept was further developed with the moral norm-activation theory of altruism (Schwartz, 1973, 1977). The norm-activation model states that individuals who believe that something 
has harmful consequences for other people or other valued objects and that they have the ability to prevent those harms, then they will feel a moral obligation to act (Schwartz, 1973, 1977; Stern et al., 1995). This theory is particularly relevant for individual choices in the COVID-19 pandemic, especially given the divergent beliefs about the severity of risks (Morning Consult, 2020) and efficacy of risk management practices (Gallup, 2021).

In addition to beliefs, values play a key role in driving decisions related to environmental harm and COVID-19 pandemic risk. Schwartz and Bilsky define values with the five key characteristics: "a) concepts or beliefs, (b) about desirable end states or behaviors, (c) that transcend specific situations, (d) guide selection or evaluation of behavior and events, and (e) are ordered by relative importance" (1995, p. 551). The Schwartz Theory of Basic Values includes ten values that have been found across many cultures: self-direction, stimulation, hedonism, achievement, power, security, conformity, tradition, benevolence, and universalism (Schwartz, 1992, 2012; Schwartz \& Sagiv, 1995). Benevolence and universalism are paired as defining a higher-order value of "self-transendence" (Schwartz, 1992, 2012; Schwartz \& Sagiv, 1995). Much of the early pro-environmental behavior literature uses the concept of self-transcendence interchangeably with altruism without distinguishing between the desire to help specific individuals and the desire to help the collective good or the biosphere (Stern et al., 1995).

Value-Belief-Norm (VBN) Theory builds on Schwartz's norm activation theory and value theory, and links it to the New Environmental Paradigm (Dunlap, Van Liere, Mertig, \& Jones, 2000), a widely used measure of an environmentalist worldview, to explain pro-environmental behavior (Stern, 2000; Stern, Dietz, Abel, Guagnano, \& Kalof, 1999). VBN theory also focuses on altruistic values as a precursor to environmental behavior (Stern, 2000). As with norm activation theory, beliefs about adverse consequences for valued objects (e.g., people or nature) and a sense of self-efficacy to reduce those threats are key pieces in the causal chain between values and action.

A separate literature on altruism has developed alongside the work on the relationship between self-transcendence and pro-environmental behavior. Interestingly, there is very little overlap between the two literatures. In 1981, Rushton and coauthors published the Self-Report Altruism (SRA) scale (Rushton, Chrisjohn, \& Fekken, 1981). This scale has been widely used, including in recent work on COVID-19 (Head, 
Kasting, Sturm, Hartsock, \& Zimet, 2020; Kasting, Head, Hartsock, Sturm, \& Zimet, 2020). The SRA scale focuses on helping behaviors like donating money or goods to charity or lending a valuable item to a neighbor. In contrast to the self-transcendence value cluster that is often referred to as altruism in the pro-environmental behavior literature, it does not measure much that could be considered collectivism, except for giving or volunteering for charities. Dietz, Fitzgerald, and Shwom (2005) is an excellent overview of the literature of environmental values and clearly lays out the development of the idea of altruism in the environmental values literature. However, there is no mention of the Rushton self-report altruism scale.

Studies that look at both NEP and SRA are sparse and we found none that directly report the relationship between these two scales (Bartczak, 2015; Randle, Kemperman, \& Dolnicar, 2019; Yuan \& Bo, 2020). There are, however, a number of studies that measure the relationship between pro-environmental measures and SRA. A study of Japanese citizens found a significant positive relationship between environmental awareness and altruism measured by the SRA scale (Aruga, 2020). In a Chinese study, researchers found that higher levels of altruism, measured by the SRA scale, led to less social discounting of environmental impacts (Guibing, Xinwei, \& Duo, 2017). Altruism, measured by the SRA, had a small positive association with green purchasing behavior, but not green purchasing attitudes (Yeoh \& Paladino, 2013). Altruism had a positive relationship with reported proenvironmental behavior and willingness to pay to protect the environment (Naderi \& Strutton, 2015). In contrast, other studies have found no relationship between altruism and pro-environmental behavior. In a study with young business school students in Australia, altruism, measured by the SRA scale, had no relationship to willingness to purchase an environmentally friendly mobile phone (Paladino \& $\mathrm{Ng}$, 2013). Also, a study of US consumers, altruism, measured by the SRA scale, had no relationship to the importance of pro-environmentally certified wood (Thompson, Anderson, Hansen, \& Kahle, 2010).

In this study, we examine the relationships between pro-environmental attitudes, altruism, and COVID-19 social distancing behavior. Using the results of an online survey of over 600 people living in the United States, we explore whether people with pro-environmental attitudes, measured by the New Environmental Paradigm (Dunlap et al., 2000), exhibit a higher willingness to reduce social exposure during the COVID19 pandemic compared to those with less pro-environmental attitudes (H1). We also 
examine whether people with higher levels of altruism, measured by the self-reported altruism scale (Rushton et al., 1981), exhibit a higher willingness to reduce social exposure (H2). Finally, we look at whether people with pro-environmental attitudes have higher levels of altruism than those with lower levels of pro-environmental attitudes (H3) and whether that altruism may drive social distancing behavior (H4).

\section{Methods}

\subsection{Survey Methodology}

Using Amazon Mechanical Turk (MTurk), we recruited 629 participants from October 21, 2020 to November 8, 2020. Amazon Mechanical Turks is an online workforce whose participants have been identified as representative of the U.S. population (Paolacci, Chandler, \& Ipeirotis, 2010), and a good source of data relative to other traditional data collection methods (M. Buhrmester, Kwang, \& Gosling, 2011; M. D. Buhrmester, Talaifar, \& Gosling, 2018). We stratified the sample to draw evenly from four US Census bureau regions (Northeast, Midwest, West, South). The survey instrument was developed with Qualtrics, an online survey platform.

We removed participants who did not complete at least $75 \%$ of the survey, leaving 612 in the sample ( $97 \%$ of the sample). These remaining observations had a median survey completion time of 23 minutes. To discard data from those who completed the survey too quickly to have plausibly read the questions, those who spent less than 8.03 minutes on the survey were removed from the sample (5th percentile completion time), leaving 581 observations. Basic demographic characteristics of the sample are summarized in Table 1.

A number of questions focused on various risk factors for serious complications from COVID-19 including age, age of household members, pre-existing conditions, and health related financial factors. Participants ranged in age from 21 to 79, with an average age of 38 ; only $3 \%$ of the sample was age 65 or older. However, $31 \%$ of the sample reported living with at least one person who was 65 or older. While we did not ask about specific conditions, $37 \%$ of the sample reported that they or another member of their household had at least one of the pre-existing conditions that the $\mathrm{CDC}$ has identified as placing them into at higher risk for serious complications from COVID-19. ${ }^{1}$ Eighty-two percent of the sample reported having health insurance and

\footnotetext{
${ }^{1}$ Including: chronic lung disease, moderate to severe asthma, serious heart conditions, compro-
} 
$83 \%$ reported having at least some paid sick leave (although a quarter of those had less than one week).

The survey was conducted at the beginning of the winter peak of COVID-19 infections. Twenty-eight participants reported having tested positive for COVID-19 (4.6\% of the sample). Fifty-seven percent of the sample reported knowing someone who had tested positive.

Given that the survey was conducted in the weeks before and days just after the 2020 election, we asked about voting intentions in the presidential race. The sample matched remarkably well with the general electorate with $48 \%$ planning to vote for Biden/Harris and $43 \%$ for Trump/Pence.

\subsection{State and Zip Code Based Data}

Using participant reported zip codes, we used the zipcodeR $\mathrm{R}$ package to pull population density data (Rozzi, 2020). The population density variable used in the models is in thousands of residents per square mile at the zip code level.

We also utilize the number of daily recorded COVID-19 cases at the state level from the CDC (for Disease Control \& Prevention, 2020). We sum the number of daily state-level cases for the week prior to the date that the participant took the survey for the participant's state of residence. Then we divide that case count by the estimated 2019 population of the state to find the per capita number of cases for the prior week (Bureau, 2019).

\subsection{Survey Response Variables \& Indices}

\subsubsection{Pro-Environmental Attitudes}

We measure pro-environmental attitudes with the New Environmental Paradigm (NEP), a widely used and validated fifteen question instrument (Dunlap et al., 2000). We sum the eight NEP items and subtract the seven Dominant Social Paradigm (DSP) items to generate a NEP value for each participant and standardize the score to have a mean of 0 and a standard deviation of 1 .

mised immune system, severe obesity $($ BMI > 40), diabetes, chronic kidney disease undergoing dialysis, liver disease 


\begin{tabular}{ll} 
Demographic Category & Percent of Sample \\
\hline 2019 Household Income & \\
Less than $\$ 25,000$ & $18 \%$ \\
$\$ 25,000$ to $\$ 49,999$ & $30 \%$ \\
$\$ 50,000$ to $\$ 74,999$ & $30 \%$ \\
$\$ 75,000$ to $\$ 99,999$ & $13 \%$ \\
$\$ 100,000$ or more & $8 \%$ \\
\hline Education & \\
High school graduate & $11 \%$ \\
Some college or technical school & $19 \%$ \\
Bachelors degree & $58 \%$ \\
Post-graduate degree & $11 \%$ \\
\hline Gender & \\
Female & $39 \%$ \\
Male & $61 \%$ \\
Non-binary & $<1 \%$ \\
\hline Race and Ethnicity & \\
Asian & $5 \%$ \\
Black or African-American & $11 \%$ \\
Latino or Hispanic & $15 \%$ \\
Native American & $4 \%$ \\
White & $83 \%$ \\
\hline Political Party & $39 \%$ \\
Democrat & $34 \%$ \\
Republican & \\
Independent or Unaffiliated & \\
Democratic Socialist & \\
\hline
\end{tabular}

Table 1: Demographic characteristics of the 581 person study sample. All participants reside in the United States. Note that race and ethnicity numbers include multiple responses for many participants for multi-racial and ethnic identities. 


\subsubsection{Altruism}

We use the Self-Reported Altruism (SRA) scale to measure altruism in our participants (Rushton et al., 1981). The scale is made up of twenty questions that ask about different ways people have helped others without compensation such as donating blood and helping a stranger (see Supplemental Materials). The scale has been cited over 400 times since its publication in 1981. Participants answer whether they have never, once, more than once, often, or very often performed each act. These responses were coded to a numeric scale from 0 to 4 and those who chose "not applicable" were coded as 0 . The responses were summed and standardized.

\subsubsection{Social Exposure Index}

The social exposure index measures the extent of reported voluntary exposure to people over the past week. We exclude relatively involuntary risk factors such as household size, employment, working from home, etc.

The number of social contacts a person has outside of work and school is an important determinant of COVID-19 risk (Jarvis et al., 2020; Li et al., 2020). We asked both how many people the participant had seen socially for more than five minutes in the past 7 days, $p_{i}$ and the frequency of such social encounters, $f_{i}$. In addition to small social engagements, we asked a yes or no question about whether the respondent had engaged in a variety of behaviors, $\eta_{i j}$, that involved non-essential contact with the community at large over the past seven days using a modified version of questions from the USC Coronavirus Tracking Survey (University of Southern California Center for Economic and Social Research, 2020). To test for internal consistency among these behaviors, we estimated the Cronbach's $\alpha$. An acceptable minimum threshold for internal consistency of a scale is 0.7 (Bland \& Altman, 1997). We include the following eight behaviors in the Social Exposure Index: going to a bar, club, or other gathering place; indoor dining at a restaurant; visiting friends, neighbors, or families' houses; hosting friends, neighbors, or family at one's house; attending a party or event with more than ten people; attending a party or event with more than fifty people; going to an indoor gym, yoga studio, or fitness center; close contact $(<6 \mathrm{ft}$ ) with non-household member (Cronbach's $\alpha=0.82$ ). Following the USC survey, we asked about the perceived risk level of a series of these activities from extremely safe to extremely unsafe (University of Southern California Center 
for Economic and Social Research, 2020). We converted these responses to a numeric 5 -point scale with unsure as the middle value.

Indexed by individual $i$ and COVID-19 risk-taking activity $j, c_{i j}$ is the score for the risk-taking behavior ('c-score'), $\eta_{i}$ is a binary variable for having recently performed $j, \bar{\eta}_{j}$ is the study population mean, $\sigma_{j}$ is the population standard deviation. $\bar{\gamma}_{j}$ is the population mean of the perceived risk of a given activity on a 0 to 4 point scale:

$$
c_{i j}=\frac{\eta_{i j}-\bar{\eta}}{\sigma_{j}} * \bar{\gamma}_{j}
$$

Thus, $c_{i}$ increases with more reported risk-taking behavior over the previous week and risk-taking behaviors that are less common and perceived as more unsafe are weighted more heavily than risk-taking behaviors that are common and perceived as more safe.

To create the Social Exposure Index, $S_{i}$, we combine the c-scores with standardized values of the number of social contacts, $p_{i}$ and frequency of social contacts, $f_{i}$ and then standardize the sum. The Social Exposure Index for each individual is given by:

$$
\begin{gathered}
\chi_{i}=\sum_{j} c_{i j}+\frac{p_{i}-\bar{p}}{\sigma_{p}}+\frac{f_{i}-\bar{f}}{\sigma_{f}} \\
S_{i}=\frac{\chi_{i}-\bar{\chi}}{\sigma_{\chi}}
\end{gathered}
$$

Thus, $S_{i}$ has a mean of 0 and a standard deviation of 1 and is increasing with higher levels of social exposure.

\subsubsection{Additional Variables}

We asked about whether the participant tested positive ( $5 \%$ of the study population) or was diagnosed by a doctor as presumptive positive (additional 10\% of the study population). To understand how participants had experienced negative financial impacts of the COVID-19 pandemic, following the survey questions of Niles et al. (2020), we asked whether they had reduced income or hours, been furloughed, or lost a job at any point during the pandemic and whether they were still experiencing those losses today. We converted these into an ordered scale where losing one's job and remaining unemployed is the worst impact while having no changes to 
one's job situation is no impact. Then we standardized the 7-point scale to have a mean of 0 and a standard deviation of 1 . Over half of the study population (295 participants) reported having lost their jobs at some point during the pandemic. This oversampling of those who have experienced or are currently experiencing unemployment is likely due to the fact that MTurk is a source of income that is more likely to be utilized during periods of unemployment or underemployment. For this reason, we control for these COVID-related financial impacts in a number of our models. To better understand their financial risks of becoming ill, we ask how many days of paid sick leave they would have available to them should they contract COVID-19 and whether or not they have health insurance (University of Southern California Center for Economic and Social Research, 2020). We also asked about basic demographics, presidential voting intentions, a series of current mental health questions (University of Southern California Center for Economic and Social Research, 2020), perceived personal risk of contracting and dying from COVID-19 (University of Southern California Center for Economic and Social Research, 2020), community social norms around masks and social distancing, views on vaccines (University of Southern California Center for Economic and Social Research, 2020), trust in medical scientists (Pew Research Center, 2019), and a multiple price lottery designed to measure risk aversion (Holt \& Laury, 2002).

\subsection{Analytical Methods}

To analyze the data and test our hypotheses, we employ a series of ordinary least squares (OLS) regressions.

Indices and likert-scale variables are scaled to set the mean equal to zero and the standard deviation equal to one. This allows for more meaningful interpretation of model coefficients.

We use ordinary least squares regressions to evaluate the relationships among variables. First we examine how the three measures of self-transcendence (NEP, SRA, and MO) relate to demographic variables, voting preferences, risk preferences, and regional variables:

$$
N E P_{i}=\beta_{0}+\beta \mathbf{X}+\gamma_{j} R_{j}+\epsilon_{i}
$$

where $\mathbf{X}$ represents a vector of demographic variables and voting preferences including age (years), gender (male indicator variable), income, education, race (white indicator 
variable), ethnicity (latino indicator variable), Trump-voter indicator variable, Bidenvoter indicator variable, risk preferences (higher values indicator more risk aversion), and indicator variables for U.S. Census divisions. We repeat this model for SRA and COVID-19 moral obligation:

$$
\begin{array}{r}
S R A_{i}=\beta_{0}+\beta \mathbf{X}+\gamma_{j} R_{j}+\epsilon_{i} \\
M O_{i}=\beta_{0}+\beta \mathbf{X}+\gamma_{j} R_{j}+\epsilon_{i}
\end{array}
$$

Next using hierarchical multiple regression, we regress voluntary social exposure on a series of covariates. In the first step, we use only the self-transcendence variable (NEP, SRA, or MO). In the second step, we add demographic variables, voting and risk preferences, and a mental health status index. In the third step, we include a wide array of COVID-19 relevant variables such as household health and age characteristics, subjective probabilities of infection and death, positive COVID-19 tests, estimated norms of mask-wearing and social exposure in one's community, number of recent cases in one's state, health insurance and paid sick leave, trust in medical scientists and vaccines, and concern about COVID-19.

To understand how whether the relationship between pro-environmental attitudes and COVID-19 risk management behavior is driven by altruistic or self-transcendent values, we use the following structural equation model (SEM) to test a mediation model of direct and indirect effects between these variables (MacKinnon, Fairchild, \& Fritz, 2007):

$$
\begin{aligned}
S_{i} & =\theta_{1}+c N E P_{i}+\epsilon_{1 i} \\
S_{i} & =\theta_{2}+c^{\prime} N E P_{i}+b M O_{i}+\epsilon_{2 i} \\
M O_{i} & =\theta_{3}+a N E P_{i}+\epsilon_{3 i}
\end{aligned}
$$

where $\theta_{1}, \theta_{2}$, and $\theta_{3}$ are intercepts, $S_{i}$ is the dependent variable, $M O_{i}$ is the mediating variable, and $N E P_{i}$ is the predictor variable. From this model, we estimate the direct effect of $N E P_{i}$ on $S_{i}$ as c' and the indirect effect of $N E P_{i}$ on $S_{i}$ through the mediating variable $M O_{i}$ as $a b$. The indirect effect and direct effect sum to create the total effect, $c$. Then we estimate the confidence intervals for these effects by bootstrapping with 10,000 samples. 


\section{Results}

We use OLS regression to examine how individual level and regional variables correlate with pro-environmental attitudes, self-reported altruism, and COVID-19 moral obligation (Table 3 ). Compared to third party voters and non-voters, proenvironmental attitudes are less strong among Trump voters $(p=0.001)$, while self-reported altruism is higher among Trump voters $(p<0.001)$. Biden voters have stronger pro-environmental attitudes $(p=0.013)$ and and no strong difference in levels of self-reported altruism $(p=0.068)$. A Wald test of coefficients shows that Trump voters and Biden voters differ on both pro-environmental attitudes $(p<0.001)$, self-reported altruism $(p=0.004)$, and COVID-19 moral obligation $(p<0.001)$. Males score lower on pro-environmental attitudes than females and other genders $(p=0.001)$ but do not differ on self-reported altruism $(p=0.769)$. There are no major correlations between the dependent variables and race, but those who identify as Latino report a stronger sense of moral obligation to protect others from COVID-19 risk than do non-Latinos $(p=0.011)$. Higher levels of risk aversion correlate with higher levels of pro-environmental attitudes $(p<0.001)$ and lower levels of self-reported altruism $(p<0.001)$. Higher levels of education correlate with higher self-reported altruism $(p<0.001)$ and has no relationship with pro-environmental values $(p=0.147)$. There are some weak regional differences in pro-environmental values and moral obligation, but compared to the East North Central division, both New England and the Pacific divisions have higher levels of self-reported altruism ( $p=0.017$ and $p=0.006$ respectively). 


\begin{tabular}{ll}
\hline Variable of Interest & Reported Behavior(s) or Opinion(s) \\
\hline $\begin{array}{l}\text { Social Exposure Index, } S_{i} \\
(\alpha=0.82)\end{array}$ & Number of social contacts, frequency of \\
& social engagement, hosted friends, attended \\
& a gathering with $10+$ people, a gathering \\
& with $50+$ people, a bar/club, indoor \\
& gym/yoga, indoor restaurant dining, \\
& neighbor/relative/friends' house \\
& Widely-used measure of pro-environmental \\
& orientation (Dunlap et al., 2000). \\
New Environmental Paradigm, $N E P_{i}$ & Widely-used measure of past altruistic \\
$(\alpha=0.85)$ & behavior with 20 questions about directly \\
Self-Reported Altruism Scale, $S R A_{i}$ & helping a stranger or acquaintance. \\
$(\alpha=0.91)$ & (Rushton et al., 1981). \\
\hline
\end{tabular}

Table 2: Table of indices created and used in the analysis of the survey data. 


\begin{tabular}{|c|c|c|c|}
\hline & \multicolumn{3}{|c|}{ Dependent variable: } \\
\hline & $\mathrm{NEP}^{z}$ & $\mathrm{SRA}^{z}$ & Moral Obligation $^{z}$ \\
\hline & $(1)$ & $(2)$ & $(3)$ \\
\hline Age (years) & -0.0002 & 0.005 & 0.004 \\
\hline Male $^{d}$ & $-0.251^{* * *}$ & -0.023 & -0.006 \\
\hline Income (1000s) & 0.001 & -0.001 & 0.001 \\
\hline Education $^{z}$ & -0.048 & $0.207^{* * *}$ & $0.066^{*}$ \\
\hline White $^{d}$ & -0.106 & 0.152 & -0.021 \\
\hline Latino $^{d}$ & 0.033 & -0.123 & $0.291^{* *}$ \\
\hline Trump-Voter ${ }^{d}$ & $-0.455^{* * *}$ & $0.490^{* * *}$ & -0.024 \\
\hline Biden-Voter ${ }^{d}$ & $0.340^{* *}$ & $0.257^{*}$ & $0.748^{* * *}$ \\
\hline Risk Aversion ${ }^{z}$ & $0.203^{* * *}$ & $-0.244^{* * *}$ & 0.002 \\
\hline East-South Central $^{d}$ & $-0.400^{*}$ & 0.075 & 0.036 \\
\hline Middle Atlantic ${ }^{d}$ & -0.062 & 0.132 & 0.151 \\
\hline Mountain $^{d}$ & -0.199 & 0.260 & $-0.285^{*}$ \\
\hline New England ${ }^{d}$ & $-0.307^{*}$ & $0.396^{* *}$ & -0.198 \\
\hline Pacific $^{d}$ & -0.183 & $0.349^{* * *}$ & -0.001 \\
\hline South Atlantic $^{d}$ & -0.075 & 0.184 & 0.230 \\
\hline West-North Central $^{d}$ & -0.023 & 0.119 & -0.021 \\
\hline West-South Central $^{d}$ & -0.220 & 0.203 & 0.179 \\
\hline Constant & $0.545^{* *}$ & $-1.658^{* * *}$ & $-0.889^{* * *}$ \\
\hline Observations & 581 & 581 & 581 \\
\hline $\mathrm{R}^{2}$ & 0.274 & 0.223 & 0.180 \\
\hline Adjusted $\mathrm{R}^{2}$ & 0.252 & 0.200 & 0.155 \\
\hline Residual Std. Error $(\mathrm{df}=563)$ & 0.865 & 0.894 & 0.919 \\
\hline F Statistic $(\mathrm{df}=17 ; 563)$ & $12.492^{* * *}$ & $9.523^{* * *}$ & $7.248^{* * *}$ \\
\hline
\end{tabular}

Table 3: OLS regressions of the New Environmental Paradigm (NEP) scale (Model 1), the Self-Report Altruism (SRA) scale (Model 2), and belief in a moral obligation (MO) to reduce COVID-19 risks (Model 3) on demographics, risk aversion, and regional variables. Dependent variables are scaled with a mean of zero and a standard deviation of one. ${ }^{*} \mathrm{p}<0.1 ;{ }^{* *} \mathrm{p}<0.05 ;{ }^{* * *} \mathrm{p}<0.01$ 


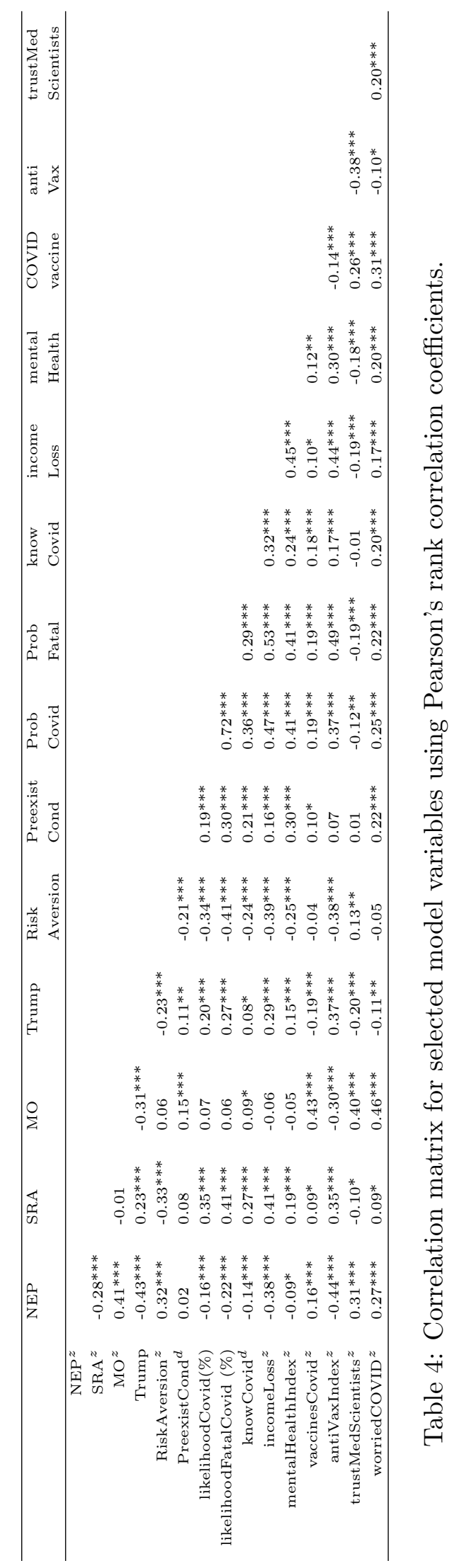


H1: People with pro-environmental attitudes have a higher willingness to adhere to COVID-19 risk reduction recommendations compared to those with less pro-environmental attitudes.

We use a series of OLS regressions to examine the relationship between NEP and voluntary social exposure (Table 5). There is a strong correlation between pro-environmental attitudes and a willingness to reduce exposure to COVID-19 by minimizing social interactions (Model $1, p<0.001$ ). This relationship persists after controlling for a wide variety of variables (Models 2 and 3). The New Environmental Paradigm (NEP) score alone explains $16 \%$ of the variation in social exposure over the past week; one standard deviation increase in the NEP score corresponds to a decrease in social exposure by $38.5 \%$ of a standard deviation.

Model 2 includes non-COVID-19 specific individual level variables - demographic variables, voting intentions, risk aversion, and mental health status (Table 5). Including these covariates slightly decreases the relationship between the NEP score and social exposure, but the relationship remains strong $(p<0.001)$. Education correlates with higher levels of social exposure $(p=0.002)$. An experimental measure of individual level risk aversion correlates strongly with decreased social exposure $(p<0.001)$ where those who are more risk averse in a lottery game appear to be more risk averse in COVID-19 exposure. A mental health index where higher values correspond with increased mental distress has a strong positive relationship with social exposure $(p<0.001)$. Trump voters exhibit higher levels of social exposure; the intention to vote for Trump correlates with $49 \%$ of a standard deviation increase in social exposure $(p<0.001){ }^{2}$ Age, gender, income, race, ethnicity, and intentions to vote for Biden do not exhibit strong relationships with social exposure in this model.

In Model 3, in addition to the variables in Model 2, a variety of COVID-19 relevant variables are included - household indicator variables regarding the presence of seniors, young children, and individuals with pre-existing conditions; subjective probabilities of contracting COVID-19 and, if contracted, dying from COVID-19; indicator variables for those who have tested positive and those who know someone who tested positive; population density in the respondent's zip code; number of

\footnotetext{
${ }^{2}$ Voting for a third party or not voting is the comparison group for the Biden and Trump indicator variables.
} 
new cases over the prior in the respondent's state; indicator variables for those who have paid sick leave and those who have health insurance; reported willingness to get the COVID-19 vaccine (standardized); an index of anti-vaccination beliefs (standardized), level of trust in medical scientists (standardized); and worry about COVID-19 (standardized) (Table 5, Model 3). The magnitude of the relationship between NEP score and social exposure is further reduced, but remains significant $(p=0.007)$. The magnitude of the relationship between voting for Trump and social exposure is also reduced, but remains significant $(p=0.011)$. Education, risk aversion, and mental health are no longer significant, indicating a correlation with additional included variables.

With the wide variety of COVID-19 relevant variables, some relationships emerged that aligned with our expectations. Others were quite surprising. For example, having already tested positive for COVID-19 is associated with increased social exposure $(p=0.034)$. Either the tendency to engage in social settings during a pandemic led to an increased likelihood of contracting COVID-19 or the positive test and the antibodies that likely follow lead to people relaxing their social restrictions. The number of new cases per capita in their state over the past week is associated with a tendency to reduce social exposure $(p=0.002)$. However, knowing someone who has tested positive for COVID-19 is also associated with increased social exposure $(p<0.001)$. Based on the concept of the availability heuristic, we would expect that knowing someone who contracted COVID-19 would lead one to believe that they may be likely to get COVID-19, yet we see the opposite relationship in the data. Perhaps if the COVID-19 case was mild, then it may have reduced their concern about the disease, leading to more risky behavior. However, this relationship could arise from social norms where people who are associating with others who are engaging in risky behavior may also be more likely to engage in risky behavior. We see further support for this explanation from the strong relationship between the estimation of whether others in their community have reduced their social gatherings compared to pre-pandemic levels with the tendency to increase one's own COVID-19exposure through optional social activities $(p<0.001)$.

Three household variables are included in model three. Having a person in one's household who has a pre-existing condition that, according to the CDC, makes them more vulnerable to complications from COVID-19 does not affect their social distancing behavior $(p=0.516)$. Having a person who is over the age of 65 in 
one's household is weakly associated with an increase in social exposure $(p=0.078)$. And having small children in one's household is associated with an increase in social exposure $(p<0.001)$. This association may arise from the extremely difficult childcare situation parents have found themselves in due to closures of schools and daycare centers during the pandemic.

We asked participants to estimate their own probability, from $0 \%$ to $100 \%$, of contracting COVID-19 in the next three months and, if they contract it, what is their estimated probability of dying from COVID-19 (University of Southern California Center for Economic and Social Research, 2020). When controlling for all other variables, the estimated likelihood of contracting COVID-19 has no relationship with social distancing behavior $(p=0.778)$; the estimated likelihood of dying from COVID-19 exhibits a weak positive relationship with social exposure $(p=0.097)$.

Participants who have experienced income loss due to the pandemic are more likely to have higher levels of social exposure $(p=0.002)$. This could be due to increased time available to socialize when one is not working or it could be a coping mechanism either psychologically or logistically. However, interaction terms between income loss and the mental health index are not statistically significant and neither is an interaction term between the mental health index and the presence of small children in one's household.

We include three variables related to the trust of vaccines and medical science. Interestingly, those who report being more likely to get the COVID-19 vaccine have more voluntary social exposure $(p=0.004)$. This indicates that social exposure may not be simply a result of rejecting the seriousness of the COVID-19 pandemic, but rather a stronger desire to engage socially with one's community making the risks more worth the benefits of social engagement. People who score high on an antivaccination sentiment index are not more or less likely to social distance $(p=0.280)$. But those who have a high level of trust of medical scientists are more likely to follow the expert recommendations and limit social exposure $(p=0.025)$.

Finally, we examined whether the relationship between NEP and voluntary social exposure would be moderated by political preferences. For Democrats, the relationship between NEP and social distancing was strong $(\beta=-0.400, p<0.001)$ with an $\mathrm{R}^{2}$ of 0.194 . For Republicans, the relationship was weaker $(\beta=-0.208, p=$ $0.053)$ with an $\mathrm{R}^{2}$ of 0.023 . Thus, while the direction of the relationship held across political affiliations, the NEP variable played a much smaller role in explaining the 
variation in social distancing behavior for Republicans than for Democrats. However, these two coefficients are not significantly different from one another at the $5 \%$ level $(p=0.097)$. Similar patterns hold when looking only at Trump voters or Biden voters.

After examining all of these different potential drivers of social distancing behavior, we find that pro-environmental attitudes are strongly associated with a tendency to limit social exposure. In the next model, we look to the role of altruism and pro-social tendencies.

H2: Pro-environmental attitudes are driven, in part, by altruistic and pro-social tendencies

The Self-Reported Altruism scale has a negative relationship with the New Environmental Paradigm $(p<0.001)$. This does not support H2. However, the belief that practicing social distancing is a moral obligation to protect others from COVID-19 strongly correlates with the NEP $(p<0.001){ }^{3}$ The NEP also strongly correlates with the worry that a family member will become seriously ill from COVID$19(p<0.001) .{ }^{4}$ Hence, the results are mixed. It appears that people with strong pro-environmental values show a high level of moral obligation to protect others from COVID-19, but they are less likely to have carried out individual-level altruistic acts unrelated to COVID-19.

H3: COVID-19 risk reducing behaviors are driven, in part, by altruistic and prosocial tendencies

In a single-variate linear regression, higher SRA scores correlate with higher social exposure $\left(\beta=0.471, p<0.001, R^{2}=0.145\right)$, which is the opposite relationship we expected. The relationship holds with non-COVID-19related control variables included in the model $(\beta=0.273, p<0.001)$, but the relationship is not associated with all COVID-related variables included in the model $(p=0.526$, Table 6 , Model $5)$.

\footnotetext{
${ }^{3}$ Moral Obligation Question: How much do you agree or disagree with the following statement: Practicing social distancing and other precautions is a moral obligation to protect those around me from COVID-19. [5-point scale from Strongly agree to Strongly disagree]

${ }^{4}$ COVID-19 Worry Question: How do you feel about the possibility that you or someone in your immediate family might become seriously ill from COVID-19 (coronavirus disease)? [Very worried, Somewhat worried, Not too worried, Not worried at all]
} 


\begin{tabular}{|c|c|c|c|}
\hline & \multicolumn{3}{|c|}{ Dependent variable: } \\
\hline & \multicolumn{3}{|c|}{ socialExposureIndex ${ }^{z}$} \\
\hline & $(1)$ & $(2)$ & $(3)$ \\
\hline $\mathrm{NEP}^{z}$ & $-0.385^{* * *}$ & $-0.220^{* * *}$ & $-0.076^{* *}$ \\
\hline $\operatorname{Age}^{d}$ & & -0.004 & -0.002 \\
\hline Male $^{d}$ & & -0.020 & 0.011 \\
\hline Income $(1000 \mathrm{~s})$ & & 0.001 & 0.001 \\
\hline Education $^{z}$ & & $0.117^{* * *}$ & 0.016 \\
\hline White $^{d}$ & & -0.086 & -0.052 \\
\hline Latino $^{d}$ & & 0.151 & -0.091 \\
\hline TrumpVoter $^{d}$ & & $0.489^{* * *}$ & $0.315^{* * *}$ \\
\hline BidenVoter $^{d}$ & & 0.054 & 0.026 \\
\hline RiskAversion $z$ & & $-0.188^{* * *}$ & -0.009 \\
\hline MentalHealthIndex ${ }^{z}$ & & $0.194^{* * *}$ & 0.032 \\
\hline PreexistCondition $^{d}$ & & & -0.027 \\
\hline SeniorInHousehold $^{d}$ & & & $0.135^{*}$ \\
\hline SmallChildrenInHousehold $^{d}$ & & & $0.112^{* * *}$ \\
\hline EstLikelihoodFatalCOVID(\%) & & & $0.003^{*}$ \\
\hline EstLikelihoodContractingCOVID(\%) & & & 0.0001 \\
\hline TestedPositiveCovid $^{d}$ & & & $0.351^{* *}$ \\
\hline KnowSomeoneWithCOVID ${ }^{d}$ & & & $0.256^{* * *}$ \\
\hline LostIncomeDuringPandemic ${ }^{z}$ & & & $0.140^{* * *}$ \\
\hline FreqMasksInCommunity(\%) & & & -0.002 \\
\hline SocialGatheringInCommunity ${ }^{z}$ & & & $0.244^{* * *}$ \\
\hline Population Density(people/sqmi) & & & $-0.004^{*}$ \\
\hline PastWeekCasesInState(cases/1000pop) & & & $-0.098^{* * *}$ \\
\hline PaidSickLeave $^{d}$ & & & 0.092 \\
\hline HealthInsurance $^{d}$ & & & $0.158^{*}$ \\
\hline LikelihoodTakingCOVIDVaccine $^{z}$ & & & $0.114^{* * *}$ \\
\hline AntiVaxIndex ${ }^{z}$ & & & 0.039 \\
\hline TrustInMedicalScientists ${ }^{z}$ & & & $-0.070^{*}$ \\
\hline WorriedAboutCOVID $^{z}$ & & & $-0.092^{* *}$ \\
\hline Constant & 0.037 & 0.030 & -0.305 \\
\hline Observations & 493 & 493 & 475 \\
\hline $\mathrm{R}^{2}$ & 0.161 & 0.356 & 0.590 \\
\hline Adjusted $\mathrm{R}^{2}$ & 0.159 & 0.341 & 0.563 \\
\hline Residual Std. Error & $0.917(\mathrm{df}=491)$ & $0.812(\mathrm{df}=481)$ & $0.640(\mathrm{df}=445)$ \\
\hline
\end{tabular}

Table 5: OLS regressions of voluntary social exposure on NEP in three models with additional covariates. Indicator variables ( 0 or 1$)$ are noted by the superscript $d$ and standardized variables, or z-scores, are noted by the superscript $z$. 
Feeling a moral obligation to practice COVID-19 precautions to protect those around them has a negative relationship with social exposure in a single variate regression but accounts for a very small proportion of the overall variation in social exposure $\left(\beta=-0.207, p<0.001, R^{2}=0.038\right)$. However, that relationship holds when all other variables are included in the model ( $p=0.050$, Table 6, Model 5$)$.

H4: The relationship between pro-environmental attitudes and COVID-19 risk reduction is mediated by altruistic and pro-social tendencies.

NEP and MO are highly correlated $(\rho=0.44)$. However, a mediation model using SEM does not show that the relationship between NEP and social exposure is mediated by a belief in a moral obligation to protect others from COVID-19 (Figure $1)$.

We use a structural equation model with bootstrapped standard errors to analyze the mediation effects. A one standard deviation increase in NEP corresponds to a $a=0.338(S . E .=0.044)$ unit increase in the belief in a COVID-19 moral obligation, where a unit corresponds to one standard deviation in the sample $(p<0.001)$. Controlling for NEP, changes in moral obligation have no effect on social exposure $(b=-0.029, S . E .=0.054, p=0.591)$. There is no indirect effect of NEP on social exposure through the moral obligation belief $(a b=-0.011, S . E .=0.021, p=0.587)$.

However, we find that the mediation runs in the opposite direction: NEP mediates the relationship between moral obligation and social exposure leaving no direct effect of moral obligation on social exposure. A one standard deviation increase in the belief in a moral obligation to protect others from COVID-19 corresponds to a $a=0.475$ $(S . E=0.054)$ standard deviation increase in NEP $(p<0.001)$. Controlling for moral obligation, an increase in NEP by one standard deviation corresponds to a change in social exposure by $b=-0.374(S . E .=0.043)$. Increases in a belief in a COVID-19 moral obligation were associated with decreases in social exposure indirectly through increases in NEP. A bias-corrected bootstrapped confidence interval of the indirect effect was below zero with a $95 \%$ confidence internal of [-0.244, -0.123]. Independent of the association with NEP, COVID-19 moral obligation is not associated with social exposure $(c=-0.029$, S.E. $=0.054, p=0.591)$. 
Table 6

\begin{tabular}{|c|c|c|c|c|c|}
\hline & \multicolumn{5}{|c|}{ Dependent variable: } \\
\hline & \multicolumn{5}{|c|}{ socialExposureIndex } \\
\hline & $(1)$ & $(2)$ & $(3)$ & $(4)$ & $(5)$ \\
\hline $\mathrm{SRA}^{z}$ & $0.273^{* * *}$ & 0.022 & & & 0.029 \\
\hline $\mathrm{MO}^{z}$ & & & $-0.109^{* *}$ & $-0.083^{*}$ & $-0.086^{* *}$ \\
\hline Age (years) & $-0.007^{*}$ & -0.002 & -0.004 & -0.001 & -0.002 \\
\hline Male $^{d}$ & 0.021 & 0.024 & 0.030 & 0.020 & 0.021 \\
\hline Income (1000s) & 0.001 & 0.001 & 0.0005 & 0.001 & 0.001 \\
\hline Education $^{z}$ & $0.067^{* *}$ & 0.013 & $0.114^{* * *}$ & 0.014 & 0.012 \\
\hline White $^{d}$ & -0.093 & -0.039 & -0.071 & -0.054 & -0.057 \\
\hline Latino $^{d}$ & 0.184 & -0.084 & 0.166 & -0.087 & -0.079 \\
\hline TrumpVoter $^{d}$ & $0.504^{* * *}$ & $0.350^{* * *}$ & $0.610^{* * *}$ & $0.344^{* * *}$ & $0.337^{* * *}$ \\
\hline BidenVoter $^{d}$ & -0.072 & 0.019 & 0.053 & 0.045 & 0.041 \\
\hline RiskAversion $^{z}$ & $-0.067^{* * *}$ & -0.007 & $-0.085^{* * *}$ & -0.008 & -0.007 \\
\hline MentalHealthIndex ${ }^{z}$ & $0.092^{* * *}$ & 0.015 & $0.100^{* * *}$ & 0.009 & 0.010 \\
\hline PreexistCondition $^{d}$ & & -0.035 & & -0.028 & -0.023 \\
\hline SeniorInHousehold $^{d}$ & & $0.148^{*}$ & & $0.154^{*}$ & $0.150^{*}$ \\
\hline SmallChildrenInHousehold $^{d}$ & & $0.114^{* * *}$ & & $0.114^{* * *}$ & $0.115^{* * *}$ \\
\hline EstLikelihoodFatalCOVID(\%) & & 0.003 & & $0.003^{*}$ & $0.003^{*}$ \\
\hline EstLikelihoodContractingCOVID(\%) & & -0.00005 & & 0.00001 & -0.00003 \\
\hline TestedPositiveCOVID $^{d}$ & & $0.348^{* *}$ & & $0.343^{* *}$ & $0.344^{* *}$ \\
\hline KnowSomeoneWithCOVID ${ }^{d}$ & & $0.263^{* * *}$ & & $0.269^{* * *}$ & $0.264^{* * *}$ \\
\hline LostIncomeDuringPandemic ${ }^{z}$ & & $0.153^{* * *}$ & & $0.157^{* * *}$ & $0.153^{* * *}$ \\
\hline FreqMasksInCommunity(\%) & & -0.002 & & -0.002 & -0.002 \\
\hline SocialGatheringInCommunity ${ }^{z}$ & & $0.248^{* * *}$ & & $0.236^{* * *}$ & $0.235^{* * *}$ \\
\hline Population Density & & $-0.004^{*}$ & & $-0.004^{*}$ & $-0.005^{*}$ \\
\hline PastWeekCasesInState (cases/1000 people) & & $-0.093^{* * *}$ & & $-0.096^{* * *}$ & $-0.095^{* * *}$ \\
\hline PaidSickLeave $^{d}$ & & 0.096 & & 0.101 & 0.093 \\
\hline HealthInsurance $^{d}$ & & $0.153^{*}$ & & $0.154^{*}$ & $0.152^{*}$ \\
\hline COVIDVaccineWilling $^{d}$ & & $0.080^{* * *}$ & & $0.093^{* * *}$ & $0.093^{* * *}$ \\
\hline AntiVaxIndex $^{z}$ & & 0.023 & & 0.020 & 0.018 \\
\hline TrustInMedicalScientists $z$ & & $-0.096^{* *}$ & & $-0.078^{*}$ & $-0.078^{*}$ \\
\hline WorriedAboutCOVID $z$ & & $-0.127^{* * *}$ & & $-0.100^{* *}$ & $-0.100^{* *}$ \\
\hline Constant & $-0.496^{*}$ & -0.029 & 0.145 & 0.160 & 0.138 \\
\hline Observations & 493 & 475 & 493 & 475 & 475 \\
\hline $\mathrm{R}^{2}$ & 0.357 & 0.586 & 0.327 & 0.589 & 0.590 \\
\hline Adjusted $\mathrm{R}^{2}$ & 0.342 & 0.559 & 0.311 & 0.563 & 0.562 \\
\hline
\end{tabular}

Table 7: OLS regressions of voluntary social exposure on altruism (SRA), moral obligation (MO), or both in five models with additional covariates. Indicator variables (0 or 1 ) are noted by the superscript $d$ and standardized variables, or z-scores, are noted by the superscript $z$. 

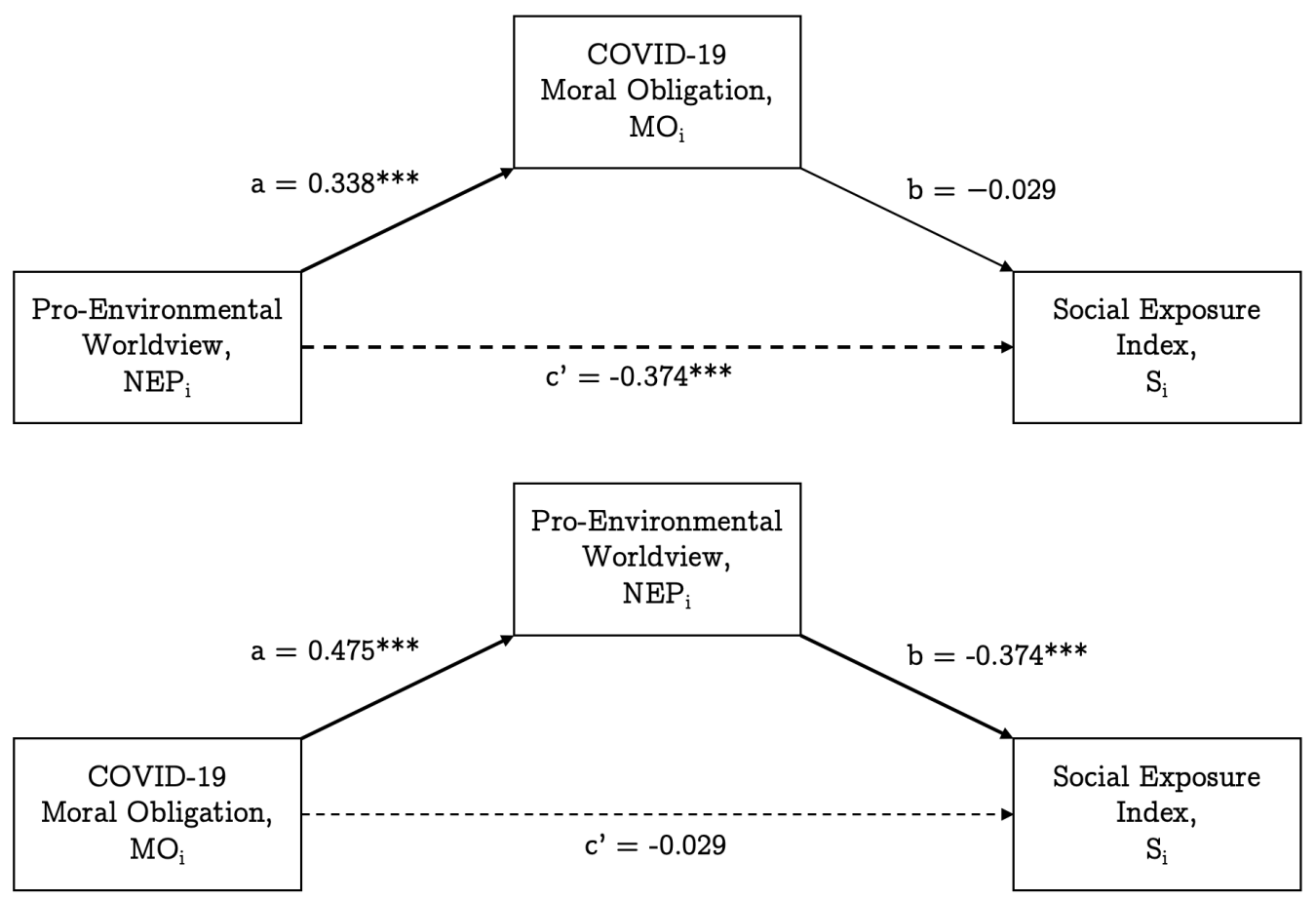

Figure 1: Mediation Analysis of the relationships between pro-environmental values (NEP), COVID-19 moral obligation (MO), and voluntary social exposure. ${ }^{*} \mathrm{p}<0.1$; ${ }^{* *} \mathrm{p}<0.05 ;{ }^{* * *} \mathrm{p}<0.01$ 


\section{Discussion}

In this study, people who have a more pro-environmental worldview, measured by the NEP, have reported less voluntary social exposure during the COVID-19 pandemic. The drivers of that finding are, however, are less clear. We will first discuss our initial hypotheses and then broaden our discussion to explore other potential drivers.

Contrary to our expectations, we also found a positive relationship between altruism, measured by the SRA scale, and voluntary social exposure. This goes against the hypothesis that people who are more inclined to help their neighbors and acquaintances are also more willing to reduce their social activities to limit the spread of COVID-19. However, when other variables related to pandemic beliefs, concerns, trust in science, and community and household characteristics are included, the relationship between altruism and social distancing disappears. Furthermore, the SRA scale has been shown to correlate strongly with extroversion (Oda et al., 2014), which could explain why those who scored higher on the SRA scale also were more likely to engage in social contact despite the risks of COVID-19 infection. For both of these reasons, we do not believe that altruism leads directly to more social exposure as is indicated by the models without COVID-19 specific control variables (Table 6 , Model 1). Instead, it is likely that SRA simply correlates with other characteristics that tend to increase voluntary social exposure during the pandemic.

A more direct question asking about the level of moral obligation that one feels to take steps to protect other people from COVID-19 shows a strong relationship with social distancing behavior. This question about the personal norm of social distancing inherently contains the norm activation theory prerequisites. In order to feel a moral obligation, one must believe that people around them are seriously threatened by COVID-19 (adverse consequences) and that actions that one could take would reduce that threat (ascription of responsibility). Given the widely varying beliefs in the United States on both the seriousness of COVID-19 for those who contract it and the effectiveness of recommended actions at reducing the spread of the disease, for a subset of the sample population, this norm would not be personally activated.

We hypothesized that the relationship between increased social distancing and pro-environmental attitudes would be driven by a tendency for people with proenvironmental attitudes to be more altruistic than those with less pro-environmental 
attitudes. This initial hypothesis is strongly supported by the literature when altruism is taken to mean that they hold self-transcendent values. Yet, we find the opposite relationship with self-reported altruism, a measure that focuses more specifically on helping behaviors and not at all on universalist values. Much of the literature connecting pro-environmental values to altruism uses universalism and benevolence interchangeably with altruism without acknowledging the separate literature on altruism as defined by Rushton (1981). Studies that focus on altruism as universalism and benevolence elicit values that align strongly with a left-leaning world view using terms that are widely rejected by right-leaning Americans, especially the type of conservatism that has developed under the leadership of Donald Trump. For example, in a simplified scale, the following three values comprise "altruistic values": "world at peace, free of war and conflict"; "Social justice, correcting injustice, care for the weak"; and "Equality, equal justice for all" (Stern, Dietz, \& Guagnano, 1998).

It appears that in this study, we are detecting the impacts of two different kinds of altruism. The first is the kind of traditional acts of face-to-face altruism where one person directly helps another. The second kind is a broader sense of moral obligation to society (i.e., collectivism or universalism). Perhaps these two versions of altruism are sometimes treated as substitutes. Culturally, the differences seem quite clear: the self-reported altruism scale focuses on acts that align with the stereotypes of small town America; it is a sort of good old-fashioned kindness. ${ }^{5}$ Conversely, a moral obligation to protect others is a value that is sometimes rejected in conservative circles. For example, a sign along a highway in Kansas that told drivers, "It's your job to keep others safe." A number of locals were outraged (Thomas, 2020). They rejected the idea that they were obligated to keep others safe even if they said that they work to help others voluntarily. The difference in approach to altruism stems in part from the choice to bestow a good act, known as autonomously motivated helping (Pavey, Greitemeyer, \& Sparks, 2012). To generalize, conservatives may value retaining autonomy over altruistic acts and thus resist claims of moral obligations, especially those coming from government policies or public servants. This insight might have predicted the backlash over COVID-19 recommendations and requirements. Even the terms that we use: "lockdown," "mask mandate," and "quarantine" all have connotations of top-down authority that may have undercut a sense of autonomy over altruistic acts of protecting one's friends and family from COVID-19.

\footnotetext{
${ }^{5}$ However, it is worth noting that the SRA had a positive relationship to population density.
} 
Those with a pro-environmental worldview may be more predisposed to cooperate with calls for collective action and less prone to backlash from calls to action by community and political leaders that seek to activate the personal norm of a moral obligation to protect people (or nature) around them. In fact, we find that a pro-environmental worldview mediates the relationship between a feeling of moral obligation to protect people around them from COVID-19 and social distancing behavior.

There are other possible explanations as to why people with pro-environmental attitudes may be more likely to social distance than those with less pro-environmental attitudes. For example, in this study, we find that people who score high on the NEP are also highly risk averse on the risk aversion spectrum as measured by Holt and Laury's (2002) multiple price lottery index (Table 3, Model 1). Risk aversion may be a common driver of both pro-environmental attitudes and social distancing behavior.

We also observed a very strong negative correlation between pro-environmental attitudes and support for Donald Trump. Likely due to the strong influence Trump had on how people viewed and responded to COVID-19 threats, Trump voters exhibit far more voluntary social exposure than non-Trump voters. In fact, other than having tested positive for COVID-19 already, reported intentions to vote for Trump for the 2020 election were the strongest predictors of social exposure in all of our models. However, neither political affiliation nor voting intentions significantly moderated the relationship between NEP and social distancing.

In future work, we will measure values directly to better understand the relationships among different types of altruism, pro-environmental attitudes, and COVID-19 risk management behavior.

\section{Conclusions}

Our study of voluntary social exposure during the COVID-19 pandemic revealed a number of insights. First, people with pro-environmental attitudes had lower levels of social exposure than people with less pro-environmental attitudes. We hypothesized that altruism might be a common thread that drives both pro-environmental attitudes and willingness to follow social distancing guidelines to reduce the spread of COVID19. We tested two different variables to understand the impact of altruism on 
social distancing: the self-report altruism scale and a feeling of moral obligation to protect others by social distancing. Self-reported altruism was not associated with voluntary social exposure, but those who feel a moral obligation to protect others have reduced their social exposure. Contrary to our expectations, the altruism score was negatively correlated with pro-environmental attitudes. Thus a tendency to exhibit individual level helping behaviors (person-to-person altruism) did not drive people with pro-environmental attitudes to increase social distancing practices. Conversely, the belief in a moral obligation to protect others from COVID-19 was positively correlated with pro-environmental attitudes. A mediation analysis indicated that the pro-environmental attitudes mediated the relationship between the belief in a moral obligation to reduce others' COVID-19 risks and social distancing behavior. While more studies are needed to draw firm conclusions, this indicates that there is a common driver of both pro-environmental attitudes and a moral obligation to protect others from COVID-19. It may be the case that holding pro-environmental attitudes predisposes one to take on additional moral obligations in collective action problems.

As the public health community seeks to find ways to reach members of the public and leverage an altruistic motivation to help those around us, it is critical to recognize that for many people, there is a substantial difference between personto-person altruism and person-to-community altruism. If we try to connect to the care that people have for those around them, then we need to know that appeals to help the overall community or the general public may fail to resonate with people whose altruistic values are motivated by the opportunity to help individual people around them. Another possible driver of these findings is the role of autonomy in altruism - many people are happy to help people around them if it is their choice to do so but resent messages that imply they are obligated to help others. More research is needed to understand specifically what kinds of messages are likely to be effective depending on one's underlying values. As we have seen during the COVID-19 pandemic, these questions have far reaching impacts for public health. Moreover, as is shown by the relationship between pro-environmental values and feelings of COVID-19 moral obligation, the need to understand how different types of altruistic appeals may resonate for some and fall flat for others can also provide new and much-needed insights to improve and broaden the effectiveness of pro-environmental communication. 


\section{References}

Aruga, K. (2020). Is environmental awareness a good predictor of an individual's altruism level? Sustainability, 12(19), 7929. doi: 10.3390/SU12197929

Bartczak, A. (2015). The role of social and environmental attitudes in nonmarket valuation. An application to the Białowiez a Forest. Forest Policy and Economics, 50, 357-365. Retrieved from http://dx.doi.org/10.1016/ j.forpol.2014.09.011 doi: 10.1016/j.forpol.2014.09.011

Batson, C. D., Ahmad, N., \& Stocks, E. (2011). Four Forms of Prosocial Motivation Egoism, Altruism, Collectivism, Principlism. In D. Dunning (Ed.), Social motivation (pp. 103-126). Psychology Press.

Bland, J. M., \& Altman, D. G. (1997, feb). Statistics notes: Cronbach's alpha. BMJ, 314(7080), 572. Retrieved from https://www.bmj.com/content/314/7080/ 572https://www.bmj.com/content/314/7080/572.abstract doi: 10.1136/ bmj.314.7080.572

Buhrmester, M., Kwang, T., \& Gosling, S. D. (2011). Amazon's mechanical Turk: A new source of inexpensive, yet high-quality, data? Perspectives on Psychological Science, 6(1), 3-5. doi: 10.1177/1745691610393980

Buhrmester, M. D., Talaifar, S., \& Gosling, S. D. (2018). An Evaluation of Amazon's Mechanical Turk, Its Rapid Rise, and Its Effective Use. Perspectives on Psychological Science, 13(2), 149-154. doi: 10.1177/1745691617706516

Bureau, U. S. C. (2019). Annual estimates of the resident population for the united states, regions, states, and puerto rico: April 1, 2010 to july 1, 2019 (nstest2019-01). https://www. census.gov/data/datasets/time-series/demo/ popest/2010s-state-total.html. (Accessed: 2020-12-16)

Dietz, T., Fitzgerald, A., \& Shwom, R. (2005). Environmental values. Annual Review of Environment and Resources, 30, 335-372. doi: 10.1146/annurev .energy.30.050504.144444

Dunlap, R. E., Van Liere, K. D., Mertig, A. G., \& Jones, R. E. (2000). Measuring Environmental Attitudes: Measuring Endorsement of the New Ecological Paradigm: A Revised NEP Scale. Journal of Social Issues, 56(3), 425-442. doi: 10.1111/0022-4537.00176

for Disease Control, C., \& Prevention. (2020). United states covid-19 cases and deaths by state over time. https://healthdata.gov/dataset/united 
-states-covid-19-cases-and-deaths-state-over-time. (Accessed: 2020$11-25)$

Gallup. (2021). Coronavirus Pandemic (Tech. Rep.). Retrieved from https:// news.gallup.com/poll/308222/coronavirus-pandemic . aspx

Guibing, H., Xinwei, Y., \& Duo, J. (2017). The effect of altruism on social discounting of environmental gain and loss. Acta Psychologica Sinica, 49(10), 1334-1343.

Head, K. J., Kasting, M. L., Sturm, L. A., Hartsock, J. A., \& Zimet, G. D. (2020). A National Survey Assessing SARS-CoV-2 Vaccination Intentions: Implications for Future Public Health Communication Efforts. Science Communication, 42(5), 698-723. doi: 10.1177/1075547020960463

Heberlein, T. A. (1972, oct). The Land Ethic Realized: Some Social Psychological Explanations for Changing Environmental Attitudes. Journal of Social Issues, 28(4), 79-87. Retrieved from http://doi.wiley.com/10.1111/j.1540-4560 .1972.tb00047.x doi: 10.1111/j.1540-4560.1972.tb00047.x

Holt, C. A., \& Laury, S. K. (2002). Risk Aversion and Incentive Effects. American Economic Review, 92(5), 1644-1655.

Jarvis, C. I., van Zandvoort, K., Gimma, A., Prem, K., Klepac, P., Rubin, G. J., ... Procter, S. (2020). Quantifying the impact of physical distance measures on the transmission of COVID-19 in the UK. BMC Medicine, 18(124), 1-10. doi: 10.1101/2020.03.31.20049023

Kasting, M. L., Head, K. J., Hartsock, J. A., Sturm, L., \& Zimet, G. D. (2020). Public perceptions of the effectiveness of recommended non-pharmaceutical intervention behaviors to mitigate the spread of SARS-CoV-2 (Vol. 15) (No. 11 November). doi: 10.1371/journal.pone.0241662

Li, W., Zhang, B., Lu, J., Liu, S., Chang, Z., Peng, C., .. Chen, J. (2020, nov). Characteristics of Household Transmission of COVID-19. Clinical Infectious Diseases, 71 (8), 1943-1946. Retrieved from https://academic.oup.com/cid/ article/71/8/1943/5821281 doi: $10.1093 / \mathrm{cid} / \mathrm{ciaa} 450$

MacKinnon, D. P., Fairchild, A. J., \& Fritz, M. S. (2007). Mediation Analysis. Annual Review of Psychology, 58, 593-614.

Menges, R., Schroeder, C., \& Traub, S. (2005). Altruism, Warm Glow and the Willingness-to-Donate for Green Electricity: An Artefactual Field Experiment. Environmental \& Resource Economics, 31 (4), 431-458. Retrieved from http:// link.springer.com/10.1007/s10640-005-3365-y doi: 10.1007/s10640-005 
$-3365-\mathrm{y}$

Morning Consult. (2020). National Tracking Poll \#2010155

October 29-31, 2020. Retrieved from https://assets

.morningconsult.com/wp-uploads/2020/11/02141747/

2010155\{_\}Crosstabs\{_\}CORONAVIRUS\{_\}CONTENT\{_\}Adults\{_\}V1\{_\}LM . $\mathrm{pdf}$

Naderi, I., \& Strutton, D. (2015). I Support Sustainability But Only When Doing So Reflects Fabulously on Me: Can Green Narcissists Be Cultivated? Journal of Macromarketing, 35(1), 70-83. doi: 10.1177/0276146713516796

Niles, M. T., Neff, R., Biehl, E., Bertmann, F., Belarmino, E. H., Acciai, F., \& Ohri-Vachaspati, P. (2020). Food Access and Food Security During COVID-19 Survey- Version 2.1. doi: 10.7910/DVN/4KY9XZ

Oda, R., Machii, W., Takagi, S., Kato, Y., Takeda, M., Kiyonari, T., ... Hiraishi, K. (2014). Personality and altruism in daily life. Personality and Individual Differences, 56(1), 206-209. Retrieved from http://dx.doi.org/10.1016/ j.paid.2013.09.017 doi: 10.1016/j.paid.2013.09.017

Paladino, A., \& Ng, S. (2013). An examination of the influences on 'green' mobile phone purchases among young business students: an empirical analysis. Environmental Education Research, 19(1), 118-145. doi: 10.1080/ 13504622.2012.687044

Paolacci, G., Chandler, J., \& Ipeirotis, P. G. (2010). Running experiments on Amazon mechanical turk. Judgment and Decision Making, 5(5), 411-419.

Pavey, L., Greitemeyer, T., \& Sparks, P. (2012). "I help because I want to, not because you tell me to": Empathy increases autonomously motivated helping. Personality and Social Psychology Bulletin, 38(5), 681-689. doi: $10.1177 / 0146167211435940$

Pew Research Center. (2019). American Trends Panel. Retrieved from https://www.pewresearch.org/wp-content/uploads/2020/03/PS_08 .02.19_trust.in_.scientists_Topline.pdf

Popp, D. (2001). Altruism and the demand for environmental quality. Land Economics, 77 (3), 339-349. doi: 10.2307/3147128

Randle, M., Kemperman, A., \& Dolnicar, S. (2019). Making cause-related corporate social responsibility (CSR)count in holiday accommodation choice. Tourism Management, 75(October 2018), 66-77. Retrieved from https://doi.org/ 
10.1016/j.tourman.2019.05.002 doi: 10.1016/j.tourman.2019.05.002

Rozzi, G. (2020). zipcoder: Data and functions for working with us zip codes. Retrieved from https://CRAN.R-project.org/package=zipcodeR (R package version 0.2 .0 )

Rushton, J., Chrisjohn, R., \& Fekken, G. (1981). The altruistic personality and the self-report altruism scale. Personality and Individual Differences, 2, 293-302. doi: 10.1016/0191-8869(81)90084-2

Schwartz, S. H. (1973). Normative explanations of helping behavior: A critique, proposal, and empirical test. Journal of Experimental Social Psychology, 9(4), 349-364. doi: 10.1016/0022-1031(73)90071-1

Schwartz, S. H. (1977). Normative influences on altruism. Advances in Experimental Social Psychology, 10(C), 221-279. doi: 10.1016/S0065-2601(08)60358-5

Schwartz, S. H. (1992). Universals in the content and structure of values: Theoretical advances and empirical tests in 20 countries. Advances in Experimental Social Psychology, 25(C), 1-65. doi: 10.1016/S0065-2601(08)60281-6

Schwartz, S. H. (1994). Are There Universal Aspects in the Structure and Contents of Human Values? Journal of Social Issues, 50(4), 19-45. doi: 10.1111/ j.1540-4560.1994.tb01196.x

Schwartz, S. H. (2012). An Overview of the Schwartz Theory of Basic Values. Online Readings in Psychology and Culture, 2(1), 1-20. Retrieved from http:// dx.doi.org/10.9707/2307-0919.1116

Schwartz, S. H., \& Sagiv, L. (1995). Identifying culture-specifics in the content and structure of values. Journal of Cross-Cultural Psychology, 26(1), 92-116. doi: $10.1177 / 0022022195261007$

Stern, P. C. (2000). Proenvironmental Consumer Behavior. Journal of Consumer Policy, 461-478.

Stern, P. C., Dietz, T., Abel, T., Guagnano, G. a., \& Kalof, L. (1999). A value-beliefnorm theory of support for social movements: The case of environmentalism. Human Ecology Review, 6(2), 81-97. doi: 10.2307/2083693

Stern, P. C., Dietz, T., \& Guagnano, G. A. (1998, dec). A Brief Inventory of Values. Educational and Psychological Measurement, 58(6), 984-1001. Retrieved from http://journals.sagepub.com/doi/10.1177/0013164498058006008 doi: $10.1177 / 0013164498058006008$

Stern, P. C., Kalof, L., Dietz, T., \& Guagnano, G. A. (1995). Values, Beliefs, 
and Proenvironmental Action: Attitude Formation Toward Emergent Attitude Objects. Journal of Applied Social Psychology, 25(18), 1611-1636. doi: 10.1111/ j.1559-1816.1995.tb02636.x

Thomas, K. (2020). Driving home from work I noticed a message... Facebook post. Retrieved from https://www.facebook.com/kristin.thomas.144/ posts/10221598529247791 (Last Accessed: 2021-02-07)

Thompson, D. W., Anderson, R. C., Hansen, E. N., \& Kahle, L. R. (2010). Green segmentation and environmental certification: Insights from forest products. Business Strategy and the Environment, 19(5), 319-334. doi: 10.1002/bse.647

University of Southern California Center for Economic and Social Research. (2020). UAS235 Wave 2 Long Form Survey Questionnaire. Retrieved from https:// uasdata.usc.edu/index.php (Understanding America Study's Understanding Coronavirus in America)

Yeoh, M., \& Paladino, A. (2013). Prestige and environmental behaviors: Does branding matter? Journal of Brand Management, 20(4), 333-349. Retrieved from http://dx.doi.org/10.1057/bm.2012.27 doi: 10.1057/bm.2012.27

Yuan, T., \& Bo, X. (2020). The Implications of SCAP Students' Personal Values and New Ecological Paradigm View on Green Behaviour: A Perspective of Ecological Man in Simplified Norm Activation Model. IOP Conference Series: Earth and Environmental Science, 576(1). doi: 10.1088/1755-1315/576/1/012033

\section{Supplementary Materials}

\subsection{New Environmental Paradigm}

Listed below are statements about the relationship between humans and the environment. For each one, please indicate whether you strongly agree, agree, neither agree nor disagree, disagree, or strongly disagree. (Source: (Dunlap et al., 2000)

1. We are approaching the limit of the number of people the earth can support

2. Humans have the right to modify the natural environment to suit their needs

3. When humans interfere with nature it often produces disastrous consequences

4. Human ingenuity will ensure that we do NOT make the earth unlivable 
5. Humans are severely abusing the environment

6. The earth has plenty of natural resources if we just learn how to develop them

7. Plants and animals have as much right as humans to exist

8. The balance of nature is strong enough to cope with the impacts of modern industrial nations

9. Despite our special abilities humans are still subject to the laws of nature

10. The so-called "ecological crisis" facing humankind has been greatly exaggerated

11. The earth is like a spaceship with very limited room and resources

12. Humans were meant to rule over the rest of nature

13. The balance of nature is very delicate and easily upset

14. Humans will eventually learn enough about how nature works to be able to control it

15. If things continue on their present course, we will soon experience a major ecological catastrophe

\subsubsection{Self-Reported Altruism Scale}

Check the category on the right that conforms to the frequency with which you have carried out the following acts. (Source: (Rushton et al., 1981).) [Never, Once, More than once, Often, Very often, Not Applicable]

1. I have helped push a stranger's car out of the snow.

2. I have given directions to a stranger.

3. I have made change for a stranger.

4. I have given money to a charity.

5. I have given money to a stranger who needed it (or asked me for it).

6. I have donated goods or clothes to a charity. 
7. I have done volunteer work for a charity.

8. I have donated blood.

9. I have helped carry a stranger's belongings (books, parcels, etc.).

10. I have delayed an elevator and held the door open for a stranger.

11. I have allowed someone to go ahead of me in a lineup (at photocopy machine, in the supermarket).

12. I have given a stranger a lift in my car for free.

13. I have pointed out a clerk's error (in a bank, at the supermarket) in undercharging me for an item.

14. I have let a neighbor whom I didn't know too well borrow an item of some value to me (e.g., a dish, tools, etc.)

15. I have bought "charity" Christmas cards deliberately because I knew it was a good cause.

16. I have helped a classmate who I did not know that well with a homework assignment when my knowledge was greater than his or hers.

17. I have before being asked, voluntarily looked after a neighbor's pets or children without being paid for it.

18. I have offered to help a handicapped or elderly stranger across a street.

19. I have offered my seat on a bus or train to a stranger who was standing.

20. I have helped an acquaintance to move households.

\subsubsection{COVID-19 Risk Behaviors}

In the last seven days, other than for essential shopping, work, and childcare, how many people outside of your household have you seen socially, in person, for more than 5 minutes? [None (0), 1 (1), 2-3 (2.5), 4-6 (5), 7-10 (8.5), 10+ (10)]

In the last seven days, other than for essential shopping, work, and childcare, how often have you had in person social interactions for more than 5 minutes with 
people outside of your household? [Never (0), Once (1), 2-3 times (2.5), Daily (7), Multiple Times per Day (14)]

In the past seven days, when you've had in person social interactions with people outside of your household, how often did those interactions include the following COVID-19 precautions: [Never (0), Sometimes (1), About half the time (2), Most of the time (3), Always (4)]

1. Mask wearing

2. Distancing at least six feet

3. Staying outdoors

\subsubsection{Perceived Efficacy of Risk Reduction Behaviors}

How effective are the following actions for keeping you and those around you safe from coronavirus? [Randomize the order of items in the list] [Responses: Extremely Ineffective, Somewhat Ineffective, Somewhat Effective, Extremely Effective, Unsure]

1. Wearing a face mask or other face covering

2. Praying.

3. Washing your hands with soap or using hand sanitizer frequently

4. Seeing a doctor if you feel sick.

5. Seeing a doctor if you feel healthy but worry that you were exposed

6. Avoiding public spaces, gatherings, and crowds.

7. Avoiding contact with people who could be high-risk.

8. Avoiding hospitals and clinics.

9. Avoiding restaurants.

10. Avoiding airplanes

11. Avoiding public parks

12. Avoiding gathering indoor social gatherings 
13. Avoiding outdoor social gatherings

14. Staying at least 6 feet away from others at all times 